\title{
GEODESICS IN METRICAL CONNECTIONS
}

\author{
RICHARD S. MILLMAN ${ }^{1}$
}

\begin{abstract}
To each connection on a Riemannian manifold we define a tensor called the $Q$-tensor. We prove that two metrical connections have the same geodesics if and only if their $Q$-tensors are equal. We then show that any manifold of dimension greater than two admits many metrical connections having the same geodesics; in particular, the $Q$-tensor is a strictly weaker invariant than the torsion.
\end{abstract}

1. Statement of theorems. We shall recall some definitions following [3]. Let $M^{n}$ be a smooth $\left(C^{\infty}\right) n$-manifold and let $T_{x}(M)$ be the tangent space at $x \in M$ and $\chi(M)$ the set of all smooth vector fields over $M$. Let $g$ be a fixed Riemannian metric on $M$. Let $T^{p, q}(M)$ be the set of tensors of type $(p, q)$. Recall that a (linear) connection on $M$ is a real bilinear operator $D: \chi(M) \times \chi(M) \rightarrow \chi(M)$ such that for all $C^{\infty}$ real valued functions, $f$, (1) $D_{f X} Y=f D_{X} Y$ and (2) $D_{X} f Y=(X f) Y$ $+f D_{X} Y$. The torsion of a given connection $D$, $\operatorname{Tor}_{D} \in T^{1,2}(M)$, is given by $\operatorname{Tor}(X, Y)=D_{X} Y-D_{Y} X-[X, Y]$ where $[$,$] denotes the$ Lie bracket. A connection $D$ is called metrical (with respect to $g$ ) if:

$$
X g(Y, Z)=g\left(D_{X} Y, Z\right)+g\left(Y, D_{X} Z\right)
$$

for all $X, Y, Z \in \chi(M)$. A curve $\alpha$ is a geodesic if $D_{d \alpha / d t} d \alpha / d t=0$. Note that by a geodesic we mean a parametrized curve and not just the geometric image of the curve.

The purpose of this note is to answer the following question: When do two metrical connections have the same geodesics? The LeviCivita Theorem states that a metrical connection (hence its geodesics) is determined uniquely by specifying its torsion. We shall show that the torsion is too strong an invariant for these purposes and that the $Q$-tensor (to be defined below) is the proper invariant.

Let $\operatorname{Tor}(M)=\left\{T \in T^{1,2}(M) \mid T(X, Y)=-T(Y, X)\right.$ for all $X$, $Y \in \chi(M)\}$. For $T \in \operatorname{Tor}(M),\left\{X_{k}\right\}, k=1, \cdots, n$, an orthonormal basis of $T_{x}(M)(x \in M)$, we define $Q^{T} \in T^{1,2}(M)$ by

Received by the editors July 18,1970 .

AMS 1970 subject classifications. Primary 53C05, 53C20; Secondary 53A99.

$K e y$ words and phrases. Metrical connections, geodesics, $Q$-tensor.

1 This paper represents research completed while the author held a Cornell Special Fellowship at Cornell University. The author would like to thank Professors R. L. Bishop and H. C. Wang for their valuable comments on this paper. 


$$
Q_{x}^{T}(X, Y)=-\frac{1}{2} \sum_{k=1}^{n}\left\{g\left(T\left(X, X_{k}\right), Y\right)+g\left(T\left(Y, X_{k}\right), X\right)\right\} X_{k}
$$

where all tensors on the right-hand side are evaluated at $x$. A simple computation shows that $Q^{T}$ is globally well defined. Let $Q(M)=$ $\left\{Q^{T} \mid T \in \operatorname{Tor}(M)\right\}$ and $q$ : $\operatorname{Tor}(M) \rightarrow Q(M)$ be given by $q(T)=Q^{T}$. If $D$ is a connection on $M$ we define $Q^{D}=Q^{\text {Tor }_{D}}$ to be the $Q$-tensor associated with $D$.

TheOREM A. Let $M$ be a Riemannian manifold with metrical connections $D$ and $\bar{D}$, then $D$ and $\bar{D}$ have the same geodesics if and only if $Q^{D}=Q^{\bar{D}}$.

Let $D$ be a connection then we may define the torsion transformation $T_{Y} \in T^{1,1}(M)$ for each $Y$ by $T_{Y}(X)=\operatorname{Tor}(Y, X)$. Because the $Q$-tensor is zero for the Riemannian connection we have the following corollary due to E. Cartan [2, p. 59].

Corollary. Let $D$ be a metrical connection on the Riemannian manifold $M$, then $D$ has the same geodesics as the Riemannian (LeviCivita) connection if and only if $g\left(T_{Y} X, X\right)=0$ for all $X, Y \in \chi(M)$.

From Cartan's viewpoint, the torsion tensor of any connection $D$ splits into several components the sum of two of which is $Q^{D}[2, \mathrm{p}$. 50-52]. We may interpret this in modern language in terms of an exact sequence of vector bundles over $M$. For each $\omega \in \Lambda^{3}(M)$ let $r(\omega) \in T^{1,2}(M)$ be defined by $g(r(\omega)(X, Y), Z)=\omega(X, Y, Z)$. Clearly $r(\omega) \in \operatorname{Tor}(M)$. In classical language, $r$ corresponds to "raise an index." From Theorem A and an easy computation in local coordinates, we have:

Theorem B. $0 \rightarrow \Lambda^{3}(M) \stackrel{r}{\rightarrow} \operatorname{Tor}(M) \stackrel{Q}{\rightarrow} Q(M) \rightarrow 0$ is an exact sequence of vector bundles over $M$.

Corollary. If $D$ is a metrical connection on $M$ then there is a different metrical connection $\bar{D}$ on $M$ with the same geodesics as $D$ if and only if $\operatorname{dim} M>2$.

The corollary follows because metrical connections are determined by their torsion and $\operatorname{dim} \Lambda^{3}(M)>0$ if and only if $\operatorname{dim} M>2$.

2. Proof of Theorem A. We fix an orthonormal basis $\left\{X_{j}\right\}$ at the point $x \in M$.

Proposition 1. Let $D$ be a metrical connection and $\bar{D}$ any connection on the Riemannian manifold $M$. Let 


$$
\bar{D}_{X} Y-D_{X} Y=S(X, Y) \quad \text { and } \quad S\left(X_{i}, X_{j}\right)=\sum_{k=1}^{n} S_{i j}^{k} X_{k} \quad(\text { near } x)
$$

then $\bar{D}$ is metrical if and only if

$$
S_{i j}^{k}=\frac{1}{2}\left\{\left(\bar{T}_{i j}^{k}-\bar{T}_{j k}^{i}+\bar{T}_{k i}^{j}\right)-\left(T_{i j}^{k}-T_{j k}^{i}+T_{k i}^{j}\right)\right\}
$$

where $T_{i j}^{k}$ (resp. $\bar{T}_{i j}^{k}$ ) are the components of $\operatorname{Tor}_{D}$ (resp. Tor $\left.\bar{D}\right)$.

Proof.

$$
\begin{aligned}
\operatorname{Tor}_{\bar{D}}(X, Y) & =D_{X} Y+S(X, Y)-D_{Y} X-S(Y, X)-[X, Y] \\
& =\operatorname{Tor}_{D}(X, Y)+S(X, Y)-S(Y, X)
\end{aligned}
$$

thus

$$
\bar{T}_{i j}^{k}=T_{i j}^{k}+S_{i j}^{k}-S_{j i}^{k}
$$

By cyclicly permuting (2) we get:

$$
\begin{aligned}
& \bar{T}_{j k}^{i}=T_{j k}^{i}+S_{j k}^{i}-S_{k j}^{i}, \\
& \bar{T}_{k i}^{j}=T_{k i}^{j}+S_{k i}^{j}-S_{i k}^{j} .
\end{aligned}
$$

$(2)-(3)+(4)$ yields

$$
\bar{T}_{i j}^{k}-\bar{T}_{j k}^{i}+\bar{T}_{k i}^{j}
$$

(5) $=\left(T_{i j}^{k}-T_{j k}^{i}+T_{k i}^{j}\right)-\left(S_{j i}^{k}+S_{j k}^{i}\right)-\left(S_{i k}^{j}-S_{i j}^{k}\right)+\left(S_{k j}^{i}+S_{k i}^{j}\right)$.

We now write out the condition for $\bar{D}$ to be metrical:

$$
\begin{aligned}
g\left(\bar{D}_{X} Y, Z\right) & +g\left(Y, \bar{D}_{X} Z\right) \\
& =g\left(D_{X} Y, Z\right)+g\left(Y, D_{X} Z\right)+g(S(X, Y), Z)+g(Y, S(X, Z)) \\
& =X g(Y, Z)+g(S(X, Y), Z)+g(Y, S(X, Z))
\end{aligned}
$$

thus $\bar{D}$ is metrical if and only if

$$
g(S(X, Y), Z)+g(Y, S(X, Z))=0
$$

hence if $X=X_{j}, Y=X_{i}, Z=X_{k}$ then (6) becomes (since the $\left\{X_{j}\right\}$ are orthonormal)

$$
S_{j i}^{k}+S_{j k}^{i}=0 \quad \text { for all } i, j, k
$$


Putting (7) into (5)

$$
\left(\bar{T}_{i j}^{k}-\bar{T}_{j k}^{i}+\bar{T}_{k i}^{j}\right)-\left(T_{i j}^{k}-T_{j k}^{i}+T_{k i}^{j}\right)=S_{i j}^{k}-S_{i k}^{j} .
$$

But (7) also says $0=S_{i j}^{k}+S_{i \mathbf{k}}^{j}$ whence adding to (8) gives the result (1). Q.E.D.

We will write $Q$ for $Q^{D}$ and $\bar{Q}$ for $Q^{\bar{D}}$.

TheOREM 2. Let $M$ be a Riemannian manifold and let $D$ be a metrical connection. Let $\bar{D}$ be any connection and form $S(X, Y)=\bar{D}_{X} Y-D_{X} Y$ then $\bar{D}$ is metrical if and only if

$$
S(X, Y)=\bar{Q}(X, Y)-Q(X, Y)+\frac{\overline{\text { Tor }}(X, Y)-\operatorname{Tor}(X, Y)}{2} .
$$

Proof. From Proposition 1 , in the basis $\left\{X_{j}\right\}$ at the point $x \in M$,

$$
\begin{aligned}
& 2 S\left(X_{i}, X_{j}\right) \\
& =\sum_{k}\left\{\bar{T}_{i j}^{k} X_{k}+\left(\bar{T}_{k i}^{j}-\bar{T}_{j k}^{i}\right) X_{k}-\left(T_{i j}^{k} X_{k}+\left(T_{k i}^{j}-T_{j k}^{i}\right) X_{k}\right)\right\} . \\
& \begin{aligned}
2 S\left(X_{i}, X_{j}\right)= & \overline{\operatorname{Tor}}\left(X_{i}, X_{j}\right)-\operatorname{Tor}\left(X_{i}, X_{j}\right) \\
& +\sum_{k}\left(\bar{T}_{k i}^{j}-\bar{T}_{j k}^{i}\right) X_{k}-\sum_{k}\left(T_{k i}^{j}-T_{j k}^{i}\right) X_{k} .
\end{aligned}
\end{aligned}
$$

However, if $Q\left(X_{i}, X_{j}\right)=\sum_{k=1}^{n} Q_{i j}^{k} X_{k}$ then by definition

$$
2 Q_{i j}^{k}=-\left(g\left(\operatorname{Tor}\left(X_{i}, X_{k}\right), X_{j}\right)+g\left(\operatorname{Tor}\left(X_{j}, X_{k}\right), X_{i}\right)\right)
$$

so

$$
Q_{i j}^{k}=\frac{1}{2}\left(T_{k i}^{j}-T_{j k}^{i}\right)
$$

where the last equality follows from the skew-symmetry of the lower indices of the torsion. Combining this last result with (9) we obtain

$$
S\left(X_{i}, X_{j}\right)=\frac{\overline{\operatorname{Tor}}\left(X_{i}, X_{j}\right)-\operatorname{Tor}\left(X_{i}, X_{j}\right)}{2}+\bar{Q}\left(X_{i}, X_{j}\right)-Q\left(X_{i}, X_{j}\right)
$$

and so the result follows because $S$ is a tensor. Q.E.D.

We now prove Theorem A. Let

$$
D_{X}^{1} Y=D_{X} Y+\frac{\overline{\operatorname{Tor}}(X, Y)-\operatorname{Tor}(X, Y)}{2}
$$

then $D^{1}$ and $D$ have the same geodesics $[3$, p. 64], hence $D$ and $\bar{D}$ 
have the same geodesics if and only if $\bar{D}$ and $D^{1}$ have the same geodesics. However, since $D$ and $\bar{D}$ are assumed to be metrical, by Theorem 2 we have

$$
\bar{D}_{X} Y=D_{X} Y+\bar{Q}(X, Y)-Q(X, Y)+\frac{\overline{\operatorname{Tor}}(X, Y)-\operatorname{Tor}(X, Y)}{2} .
$$

Because the last term is skew-symmetric we have $\left[3\right.$, p. 64] $\bar{D}$ and $D^{1}$ have the same geodesics if and only if $Q(X, Y)-\bar{Q}(X, Y)$ is skewsymmetric. However, by its very definition $Q(X, Y)$ is symmetric, hence $Q(X, Y)-\bar{Q}(X, Y)$ is skew-symmetric if and only if $Q(X, Y)$ $-\bar{Q}(X, Y)=0$. Q.E.D.

The result that 2 metrical connections need not coincide in order to have the same geodesics may also be obtained from two theorems of $[1$, pp. 130-131].

\section{BIBLIOGRAPHY}

1. R. L. Bishop and R. J. Crittenden, Geometry of manifolds, Pure and Appl. Math., vol. 15, Academic Press, New York, 1964. MR 29 \#6401.

2. E. Cartan, Sur les variêtés à connexion affine et la theorie de les relativité généralisée, Ann. Sci. École Norm. Sup. (3) 42 (1925), 17-88.

3. N. J. Hicks, Notes on differential geometry, Van Nostrand Math. Studies, no. 3, Van Nostrand, Princeton, N. J., 1965. MR 31 \#3936.

Cornell University, Ithaca, New York 14850

Ithaca College, IthaCA, New York 14850 